\title{
Male Reproductive System
}

National Cancer Institute

\section{Source}

National Cancer Institute. Male Reproductive System. NCI Thesaurus. Code C12722.

The sex organs of the male. 